\title{
La dynamique de pouvoir dans la représentation de l'Autre. Le cas des arts de l'Islam en Europe
}

The Dynamics of Power in Representing the Other. The Case of Islamic Art in Europe

Obay Al Bitar

\section{(2) OpenEdition}

\section{Journals}

Édition électronique

URL : https://journals.openedition.org/iss/3015

DOI : $10.4000 /$ iss.3015

ISSN : 2306-4161

Éditeur

ICOM - International Council of Museums

Édition imprimée

Date de publication : 18 décembre 2021

Pagination : 41-55

ISBN : 978-92-9012-446-7

ISSN : 2309-1290

\section{Référence électronique}

Obay Al Bitar, «La dynamique de pouvoir dans la représentation de l'Autre. Le cas des arts de l'Islam en Europe », ICOFOM Study Series [En ligne], 49-1 | 2021, mis en ligne le 18 décembre 2021, consulté le 08 janvier 2022. URL : http://journals.openedition.org/iss/3015; DOI : https://doi.org/10.4000/iss. 3015 


\section{La dynamique de pouvoir dans la représentation de l'Autre. Le cas des arts de l'Islam en Europe.}

\section{Obay Al Bitar}

Université de Liège - Liège, Belgique.

Je suis Narcisse et je veux lire dans les yeux de l'autre

une image de moi qui me satisfasse.

Frantz Fanon

RÉSUMÉ

La discipline d'étude des arts de l'Islam est fondée au tournant du XXe siècle en Occident. Née de contacts antérieurs avec les terres de l'Islam, la fascination des érudits européens pour l'Orient a provoqué une vague d'expéditions dans le but de récolter davantage d'informations utilisées pour paver le chemin des conquêtes militaires européennes et la création des colonies dans la région. Ce nouveau monde est par la suite ramené en Europe pour être exposé et redécouvert par le public européen. Les objets exotiques du monde conquis ont été soumis à des représentations qui renforcent la séparation et la domination 
raciale et culturelle. L'analyse des expositions permanentes des arts de l'Islam en Europe démontre d'une volonté de créer des ponts entre les civilisations contemporaines et d'un effort d'adoucissement de la mémoire d'un passé colonial en offrant ainsi un regard sur la pratique consistant à exposer la culture de l'Autre.

Mots clés: muséographie, scénographie, orientalisme, colonialisme, Orient, Occident.

\section{ABSTRACT}

\section{The Dynamics of Power in Representing the Other. The Case of Islamic Art in Europe.}

The discipline of the study of Islamic Art in the West was founded at the turn of the $20^{\text {th }}$ century. Born out of previous contact with the lands of Islam, European scholars' fascination with the Orient prompted a wave of information-gathering expeditions to the Middle East that later paved the way for European military conquests and the establishment of colonies in the region. The discovered new world was then brought back to Europe to be rediscovered by the European public through permanent exhibitions. Exotic objects from the conquered world have been subjected to representations that reinforce racial and cultural separation and domination. An analysis of present-day permanent exhibitions of Islamic Art in Europe not only reveals a desire to build bridges between contemporary civilizations in an effort to soften the memory of the colonial past but also informs the practice of exhibiting the cultural heritage of the Other.

Key words: museography, scenography, orientalism, colonialism, Islamic art, Europe.

\section{$\star$}

\section{Introduction}

Le début du $\mathrm{XXI}^{\mathrm{e}}$ siècle marque une réévaluation importante dans le champ d'étude de l'art et de l'archéologie islamique. L'évolution scientifique dans le domaine des arts de l'Islam ou Islamic art ainsi que les récents changements socio-politiques notamment dans le monde de l'Islam ont inspiré une modification de notre regard sur cette discipline. Cela est notamment illustré à travers des musées qui, pour la plupart d'entre eux, ont été ou sont encore en cours de réorganisation de leurs collections des arts de l'Islam. Par exemple, le Benaki Museum à Athènes ouvre sa section d'Art Islamique en 2004, suivi par 
l'ouverture du Museum of Islamic Art à Doha en 2008 et par la réouverture du David Collection Museum en 2009 suite à d'importants travaux de rénovation. En 2010, le premier musée des arts de l'Islam rouvre ses portes au Caire et le Musée d'Art Islamique du Pergamonmuseum fait l'objet de travaux de rénovation qui devront s'achever en 2024. Des petites collections sont aussi ouvertes au public dont la galerie d'Art du monde islamique au Musée du Cinquantenaire à Bruxelles ouverte depuis 2008, et les galeries Arts of the Islamic World ouvertes à partir de l'année 2009 au Brooklyn Museum.

Le changement et le développement du domaine se reflètent également dans le nom des nouvelles galeries des musées. Par exemple, la galerie des arts de l'Islam au Victoria \& Albert Museum ouverte en 2006 est attribuée au nom de son mécène principal, Jameel Gallery of Islamic Art'. C'est aussi le cas au British Museum avec l'Albukhary Foundation Gallery of the Islamic world, ouverte depuis $2018^{2}$. Cependant, le Metropolitan Museum of Art à New York a choisi d'attribuer le nom de ses galeries selon l'emplacement géographique des collections. Ses nouvelles galeries sont effectivement appelées Arts of the Arab Lands, Turkey, Iran, Central Asia and Later South Asia et sont ouvertes depuis 2011. En revanche, le musée du Louvre s'est approprié l'utilisation du terme arts de l'Islam dans ses nouvelles salles ouvertes en 2012 et caractérisées par une intervention architecturale contemporaine afin de suggérer une ambiance orientale ${ }^{3}$. De manière similaire, les arcs en ogive et les colonnes engagées employés dans la galerie permanente des arts de l'Islam au Detroit Institute of Arts, ouvert en février 2010, font référence à la mosquée d'Ibn Tulun à Caire. En effet, l'utilisation des éléments et des motifs architecturaux tridimensionnels comme le moucharabieh présent dans la plupart des musées des arts de l'Islam, contribuent à une mise en contexte en attachant les objets à un espace et/ou à un lieu spécifique. L'exposition des objets des arts de l'Islam dans une ambiance romanesque jugée authentique d'un point de vue occidental, se caractérise par un désintérêt pour l'orient actuel en faveur d'un retour au passé et une curiosité pour l'exotisme. Développé à l'époque coloniale, ce modèle de représentation se situe dans une continuité des pratiques d'exposition des arts non-occidentaux pour marquer la séparation et la domination de l'Occident sur l'Orient (Saïd, 2005).

\footnotetext{
I. Mohammed Abdul Latif Jameel s'est impliqué en 2003, dans le projet de rénovation des espaces consacrés aux arts de l'Islam au Victoria \& Albert Museum (Crill \& Stanley, 2006). Il a également fondé le prix Jameel pour l'art contemporain qui a pour objectif d'explorer la relation entre les pratiques des arts de l'Islam et les arts occidentaux dans l'art contemporain.

2. L'Albukhary Foundation Gallery of the Islamic world est créée grâce à la donation de Syed Mokhtar Albukhary, président de l'Albukhary Fondation, ainsi qu'au soutien de Syed Mohamed Albukhary, directeur de l'Islamic Arts Museum Malaysia (IAMM) (Greenwood, 2019). L'intention de ces mécènes porte sur la création d'un dialogue entre le monde de l'Islam et l'Occident.

3. Le terme oriental désigne tout d'abord une frontière géographique, appliquée à l'Asie, et il comprend un regard sur toute sa capacité et ses caractéristiques ethniques, morales et culturelles. Il renvoie à une notion d'exotisme, du mystérieux et du profond et dénote d'une passion de tout ce qui est asiatique (Said, 2005).
} 
Quel regard pouvons-nous porter sur ce changement frappant le monde muséal? Quel est l'intérêt porté par les musées dans leurs expositions des arts de l'Islam et comment cela peut-il traduire une réflexion sur leurs propos? Enfin, quels sont les enjeux dissimulés derrière la contextualisation scénographique évoquant une ambiance orientale à caractère essentialiste ? À travers l'analyse de trois musées européens ${ }^{4}$, à savoir le Pergamonmuseum, le musée du Louvre et le British Museum, nous tenterons de mettre en lumière la conception des expositions permanentes des arts de l'Islam pour nourrir une réflexion sur la conception des expositions des arts non-occidentaux en Europe et en Amérique de nord. Ce travail de recherche a pour but d'offrir un regard sur les problèmes liés aux expositions de la culture de l'Autre, et notamment celles des arts de l'Islam. Ce modèle d'exposition occidental caractérisé par l'eurocentrisme façonne la perception et la conception esthétique de la mémoire visuelle du monde de l'Islam. Afin de le déconstruire, nous allons retracer le développement historique de la relation entre l'Orient et l'Occident tout en offrant un aperçu des problèmes d'une discipline mal définie.

\section{Terminologie et définition}

L'art produit sur les terres de l'Islam a été défini par plusieurs adjectifs qui se fondent autour des peuples, religions, et races projetant ainsi une vision

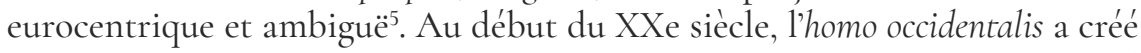
la notion d'art et d'architecture dite islamiques ${ }^{6}$, afin de projeter une identité religieuse englobant le monde moderne. Ce terme sert à mettre en lumière l'unité présumée de l'art dans les régions où les populations majoritaires qui y vivent pratiquent la foi musulmane. D'un point de vue occidental, l'adjectif islamique aide à rattacher l'art qu'il définit à des notions normatives comme le christianisme ou le judaïsme, mais par cette simplification les variétés hétérodoxes comme le chiisme, le sunnite ou le soufisme sont négligées. Ainsi, l'unité cherchée dans les arts de l'Islam ne sert qu'à expliquer les similitudes entre différentes productions artistiques afin de simplifier les cas de parallélisme distingués dans l'histoire de l'art de l'Islam (Shalem, 2012). Dans ce sens, le style et le langage esthétique sont détachés de l'effort produit par une culture

\footnotetext{
4. Ces trois musées diffèrent dans leur finalité. Le Pergamonmuseum à Berlin est un musée d'art et d'archéologie tandis que le musée du Louvre à Paris est un musée d'Histoire de l'art. Quant au British Museum à Londres, il s'agit d'un musée multidisciplinaire avec une approche basée sur la culture matérielle.

5. Edward Said (2005) parle de la notion d'Oriental en expliquant se référer à la race. Les termes tels qu'art arabe, art turc et art persan sont employés à l'exposition universelle à Paris en 1893 et mettent l'accent sur la race. Pour Sophie Makariou (Labrusse, 2007), le terme « arabe » désigne des populations arabisées.

6. Selon Nasser Rabbat (2004), les études des arts de l'Islam ont été encadrées dans les théories de l'art occidental qui trouvent ses racines à la fin du XVIIIème siècle. Pour lui, ces théories rendent ces études conditionnées à une hiérarchie prescrite de manière chronologique, géographique et même idéologique. Rabbat précise que l'intérêt pour l'architecture islamique remonte aux années I820 suite aux campagnes militaires par Bonaparte.
} 
dans une période et un espace spécifiques et peuvent être facilement transférés dans l'espace et le temps (Allen, 1988) ${ }^{7}$. De même, certains objets créés par des musulmans peuvent servir à la religion, mais la majorité des objets sont séculaires. D'ailleurs, le terme Islamic est utilisé pour renvoyer à la culture des pays dans laquelle l'islam joue un rôle dominant sans être unique dans la foi (Grabar, 1973). Dans cette interprétation, le terme Islamic Art sert à couvrir l'intégralité du champ des pays d'Islam (Labrusse, 2007, p. 196). Blair et Bloom (2003) associent le terme à la culture visuelle d'un temps et d'un espace où l'islam en tant que système politique règne. De plus, une nouvelle terminologie est proposée afin de définir les cultures visuelles de l'islam comme Les arts des terres de lislam, ce qui pourrait constituer une solution (Shalem, 2012, p. 14). Enfin, il est nécessaire de distinguer le mot islam qui renvoie à la religion et le mot Islam qui renvoie à la civilisation ${ }^{8}$. Dès lors, le terme arts de l'Islam est le meilleur compromis concernant le problème de la terminologie.

Les arts de l'Islam désignent donc l'ensemble du langage visuel se situant sur un vaste territoire dont le système politique dominant est l'islam?. Alors que la religion islamique est présente dans des régions d'Afrique, d'Europe de l'est, du sud de la Russie, de l'ouest de Chine, du nord de l'Inde et du sud-est de l'Asie, ses frontières géographiques actuelles couvrent l'Asie de l'ouest et s'étendent jusqu'à la côte atlantique de l'Afrique du nord ainsi que l'Afrique sub-saharienne et l'Espagne jusqu'à l'Asie centrale et l'est de l'océan Indien. Par ailleurs, les études des arts de l'Islam se focalisent sur le cour de la terre islamique entre l'Égypte et l'Asie centrale du VIIe au XVIIIe siècle, en passant occasionnellement par des incursions en Espagne, en Sicile et en Inde (Blair et Bloom, 2003). Bien que la majorité des musées et des historiens limitent les arts de l'Islam à une période précise (jusqu’à la fin de l'Empire ottoman au XIXe siècle), il semble que l'intégration de l'art contemporain dans le discours de la discipline et notamment dans le discours muséal soit préférable ; isoler l'art contemporain impliquerait une date déterminée aux civilisations de l'Islam privant l'histoire contemporaine de faire partie de la civilisation islamique. De plus, cette date d'expiration imposée par l'Occident est mise en concordance avec la création de la notion de l'Orient. Le risque de cette décision arbitraire est de tomber dans le piège de l'orientalisme ${ }^{10}$. Cette limitation dans le discours historique

7. Sheila S. Blair et Jonathan M. Bloom (2003) trouvent que l'architecture ottomane a très peu à voir avec l'architecture iranienne et qu'il est très difficile de trouver des similitudes significatives entre l'Alhambra et le Taj Mahal.

8. D'après un entretien d'Obay Al Bitar avec Gwenaëlle Fellinger, la conservatrice en chef du département des arts de l'Islam au musée du Louvre, Paris, le 28 novembre 2018.

9. Nous avons choisi d'identifier les arts de l'Islam selon leurs critères visuels afin d'appuyer le rejet des normes imposées en Occident. Les arts de l'Islam ne peuvent pas être comparés aux formes d'art européen et demandent un traitement éloigné des approches eurocentrées. Notre proposition ne constitue en aucune manière une solution innovante, définitive. Tout au plus ouvre-t-elle le questionnement sur une discipline qui est sans doute mal définie et nécessite encore un travail de réévaluation.

IO. L'orientalisme selon Edward Said (2005, p. I4I), est la discipline dans laquelle l'Orient est abordé 
de la discipline des arts de l'Islam pose donc une difficulté certaine pour la compréhension de la civilisation de l'Islam en Occident. Oleg Grabar (1983) associe ce problème aux ouvrages abordant les productions artistiques et les monuments architecturaux créés jusqu’à la fin du XVIIe siècle, notamment dans des régions comme le Levant, l'Égypte, l'Iran et l'Asie centrale. Selon lui, cette approche est problématique parce qu'elle néglige d'autres régions où réside une large communauté musulmane. Elle indique du même coup que les arts et les civilisations de l'Islam ont cessé d'exister il y a deux cents ans. Enfin, elle suggère que seul le lointain peut être appelé art de l'Islam. Les formes d'art qui ont survécu - que cela soit dans le domaine de la musique de l'architecture ou même des arts plastiques et de la calligraphie $(\mathrm{Ali}, 1997)$ - assurent néanmoins la continuité de la civilisation islamique.

\section{Les arts de l'Islam et l'Europe}

Les contacts entre l'Europe occidentale et le monde de l'Islam tout au long du XIX ${ }^{e}$ siècle sont basés principalement sur l'impérialisme et le colonialisme. Ces doctrines politiques ont redéfini la structure du pouvoir mondial et sont motivées par un intérêt économique pour le monde non-occidental riche en matières premières et en main-d'œuvre (Çelik, 1992). Mais le monde oriental a également fait l'objet d'une fascination intellectuelle suscitée par des textes et des études, des artefacts culturels, des objets ethnographiques et des structures urbaines (Maussen, 2007). À travers les musées, nous allons élaborer ce rapport de force en évoquant le cas célèbre du South Kensington Museum, qui a été construit suite à la grande exposition industrielle de Londres de 1851.

Ce musée d'art et d'industrie, établi en 1852 et renommé en 1899 le Victoria \& Albert Museum, est ouvert au public sur son site actuel en 1857 avec pour but d'augmenter les moyens de formation industrielle et d'étendre l'influence de la science et de l'art sur l'industrie productive (Robertson, 2004). À l'époque, les objets non-occidentaux servent d'exemple au design idéal pour la fabrication industrielle et une source d'inspiration pour les producteurs et les ouvriers dans le marché britannique. La collection récoltée depuis 1850 en provenance d'Inde et d'Asie du sud-est, de Chine et du Japon, représente un microcosme d'une vision moderne de monde en miniature (Conway, 1882). Dans son analyse, Tim Barringer (1998) voit dans la scénographie et la technique d'affichage dans les salles où les objets non-occidentaux sont exposés une volonté d'équilibrer le

en tant que sujet d'étude. Elle inclut l'édition et la traduction des textes mais également les études numismatiques, anthropologiques, archéologiques, sociologiques, économiques, historiques, littéraires et culturelles dans toutes les civilisations connues de l'Asie et de l'Afrique du nord, anciennes et modernes. Ce terme est aussi utilisé pour désigner la collection de rêves, dimages et de vocabulaires employés pour parler de ce qui se trouve de l'autre côté des frontières. Pour Avinoam Shalem (20I2), l'orientalisme est une approche méthodologique et critique qui sert en premier lieu à réexaminer l'influence du mode de pensée eurocentrique qui est à la base de la connaissance de l'époque sur l'Orient, afin d'en réévaluer la compréhension et d'obtenir une image plus précise des contrastes existant sur cet immense territoire. 
processus d'éducation et le divertissement populaire, tout en fournissant une source d'inspiration via les décorations indiennes, islamiques et japonaises. Selon lui, cette ambiance orientale dans laquelle les objets non-occidentaux sont regroupés et isolés de ce qui était le courant dominant de l'époque sert l'objectif orientaliste. Ainsi, l'accent est mis sur l'isolement de ces objets en marquant leur différence, ce qui renforce la séparation entre l'Orient et l'Occident et affirme la domination de l'Orient par l'Occident, tout en estompant les différences au sein des catégories orientales (Said, 2005) ${ }^{11}$. Dès lors, cette stratégie d'exposition symbolise la base d'une hiérarchie coloniale et représente une idéologie de supériorité raciale et culturelle sur l'Orient. En effet, les objets sont exposés pour leur importance politique et deviennent le symbole de la victoire de l'empire britannique.

Ce mouvement de célébration des triomphes économiques et industriels occidentaux est notamment illustré à travers les expositions universelles du XIXe siècle en Europe et en Amérique du nord. En effet, les expositions universelles tentent de représenter le progrès technologique de l'Europe et de mettre en valeur les bénéfices retirés des colonies (Fulco, 2017). Elles ont cherché à recréer une ambiance authentique ancrée dans la perception d'occidentaux depuis longtemps, sur un monde monothéiste, de fantasme et d'exotisme, en bâtissant des répliques de monuments connus et en exposant l'Homme dans son environnement ethnique. En introduisant une vision du monde en microcosme, elles offrent un voyage imaginaire dans un nouveau cadre de tourisme via l'introduction d'éléments orientalistes dans la représentation des arts non-occidentaux ${ }^{12}$. Dès lors, elles déterminent une hiérarchisation qui définit la catégorisation de l'art non-occidental. De plus, des représentations authentiques des coutumes et des habitudes des cultures étrangères sont ajoutées à l'expérience offerte par l'exposition. Cela conduit à la construction de structures indigènes pour accueillir les différentes variétés ethniques au monde (Leprun, 1986). Les expositions universelles ont également eu une influence sur la politique d'acquisition, sur le mode d'affichage et la présentation des objets non-occidentaux dans les musées en Europe. Elles ont laissé un héritage d'une muséographie orientaliste fondée sur des représentions pittoresques, articulées sur la préférence d'une accumulation des objets de manière désordonnée plus que sur l'exclusivité de l'objet individuel (Roxburgh, 2000). En revanche, l'exposition Meisterwerke muhammadanischer Kunst à Munich en 1910, qui présente

\footnotetext{
II. Selon Said (2005, p. 96), on peut se servir de l'Orientalisme pour renforcer l'autorité et la séparation et pour exprimer la force de l'Occident et la faiblesse de l'Orient, telles que les voit l'Occident.

I2. Des répliques des monuments trouvés à l'Alhambra ont été recréées et exposées au Crystal Palace en $185^{1}$; les tribaux architecturaux créés au South Kensington Museum illustrant la porte de Sanchi en Inde ont été reproduits et exposés dans des expositions universelles en France et en Allemagne (Barringer, I998) ; une version des rues du Caire a été créée aussi lors de l'Exposition universelle de 1889 à Paris ; etc. Ils ont ainsi introduit les cultures comme des artefacts exposés dans des pavillons qui étaient eux-mêmes des résumés des cultures (Çelik, I992).
} 
les chefs-d'œurre dans des vitrines isolées va marquer la muséographie des arts de l'Islam jusqu'à nos jours' ${ }^{13}$.

\section{La muséologie des arts de l'Islam aujourd'hui en Europe}

Lors de nos observations et analyses des différents musées occidentaux, ces derniers ont fait l'objet d'un renouvellement de leur parcours, en allouant plus d'espace et d'importance aux expositions de collections des arts de l'Islam. Ce changement est notamment dû au désir de rétablir un pont entre les cultures occidentales et islamiques. C'est le cas à Londres et à Paris, par exemple, où les collections des arts de l'Islam ont été constituées dans un pays étranger à leurs pays d'origine. Ces collections ont été exposées auparavant comme un symbole du pouvoir colonial et impérialiste au XIX et début XX $X^{\mathrm{e}}$ siècle. Ce temps est maintenant révolu, et l'existence de ces collections a encouragé les musées à créer de nouvelles salles d'exposition ainsi qu’à prendre la responsabilité de conserver et d'exposer les cultures non-occidentales. Giovanni Pinna (2011) affirme par ailleurs que les changements de présentation des arts de l'Islam dans les musées en Occident servent à adoucir le passé colonial et portent un intérêt politique pour le monde de l'Islam. Dans les parties qui suivent, nous focaliserons notre analyse sur l'approche de trois musées : le Pergamonmuseum à Berlin, le musée du Louvre à Paris et le British Museum à Londres. Le choix de ces trois villes vient de leur importance dans le développement de la discipline des arts de l'Islam. En outre, ces trois musées représentent des établissements majeurs dans la présentation des cultures et des arts non-occidentaux.

\section{Notion des arts de l'Islam}

Le Pergamonmuseum, tel qu'on le visite aujourd'hui, présente les collections d'art antique classique - Antikensammlung, du musée d'Antiquité du Proche-Orient - Vorderasiatisches Museum et du Musée d'Art Islamique - Museum für Islamische Kunst. Il expose principalement des objets archéologiques issus des campagnes de fouilles dans les régions de la Méditerranée et du Proche-Orient effectuées à partir de 1875. L'intérêt allemand de construire un Musée d'Art Islamique date de 1904 avec la création du département d'art antique islamique au sein du Kaiser-Friedrich-Museum par Wilhelm von Bode (1845-1908) (Junod et al., 2012). Le département est créé grâce à l'arrivée de chefs-d'œuvre en Allemagne après les fouilles dans les territoires de l'Empire ottoman au Moyen-Orient et les Kadjars (1779-1925) en Iran. Cela témoigne des bonnes relations diploma-

\footnotetext{
I3. Les musées d'art et d'archéologie accordent une importance à l'exposition d'un objet seul sous prétexte d'un Meisterwerk. Les chefs-d'œuvre sont considérés comme des éléments muséographiques importants pour attirer les visiteurs et les inviter à se rendre au musée, notamment au musée du Louvre où les objets exposés renvoient à leurs matérialités et à leurs valeurs esthétiques. Le British Museum s'articule, quant à lui, dans sa présentation sur les objets de la vie de tous les jours. Son but n'est pas de présenter les objets de luxe, fabriqués pour les élites dirigeantes, mais d'exposer la réalité des sociétés du monde islamique à travers des objets réels de la vie quotidienne.
} 
tiques entre ces deux empires et l'Allemagne. Ensuite, le département devient le Museum für Islamische Kunst ouvert au Pergamonmuseum en 2001. Le Pergamonmuseum fait partie d'un grand complexe muséal conçu sur l'île des Musées. Le projet, envisagé depuis 1841 par le Roi Friedrich Wilhelm IV (1795-1861) est toujours en cours de réalisation. Ce complexe muséal est destiné à devenir une ville temple de l'art et de la culture de 6000 ans d'histoire humaine (Junod et al., 2012, p. 294 $)^{14}$. Dès lors, le Pergamonmuseum offre dans son ensemble un parcours reliant l'Antiquité classique et proche-orientale avec les arts de l'Islam. Le complexe muséal sur lî̂le aux Musées va ensuite permettre de faire le lien entre les antiquités méditerranéennes et proche-orientales avec l'évolution de l'art en Occident. Ce parcours offre une image complète des développements des arts en Europe ainsi qu'une mise en évidence de la relation entre l'Europe et les régions de la Méditerranée et du Proche-Orient.

Le musée du Louvre à Paris est un musée universel composé de huit départements mettant en valeur l'histoire de l'art - en premier plan - et l'archéologie. Les œuvres exposées au musée du Louvre sont présentées dans un ordre chronologique, de l'Égypte pharaonique et la Grèce antique jusqu'à l'art occidental du XIX ${ }^{e}$ siècle. L'exposition permanente des arts de l'Islam, ouverte en 2012, offre une continuité du discours muséographique du musée situant les arts de l'Islam entre les antiquités de la Grèce préclassique et de l'Égypte copte et romaine d'un côté, et de l'Orient méditerranéen à l'époque romaine de l'autre. La mise en place d'un département complètement dédié aux arts de l'Islam se fait en 2003. Le choix d'établir ce département est tout d'abord un choix politique fait par le président de la République Jacques Chirac (1932-2019). Jean-Loup Amselle (2016, pp. 24-25) voit dans ce choix une volonté de faire plaisir aux pays musulmans dont certains ont financé ce projet (Maroc, pays du Golfe) ainsi qu'aux musulmans de France. La création du département s'est accompagnée d'une autonomisation de la collection au sein du Louvre et de la réalisation d'un nouvel espace architectural en 2012. Ce département affirme également l'importance scientifique accordée par la ville de Paris au développement de la discipline des arts de l'Islam. Depuis la formation de la collection d'art persan en 1893, et la première salle des arts musulmans au musée du Louvre en 1905 suivie par une salle d'art islamique en 1993 (Makariou, 2012), ce changement témoigne de l'évolution d'un siècle d'études et de recherches sur la discipline. Les arts de l'Islam au Louvre sont passés d'un art ethnique à un art religieux pour ensuite désigner au XXI e siècle l'art des civilisations où les élites dirigeantes pratiquent la foi musulmane.

Enfin, la naissance de la collection des arts de l'Islam au British Museum a commencé avec la construction du musée suite au legs de Sir Hans Sloane (1660-1753) (Duthie, 2011). Il avait dans sa collection une partie des objets

\footnotetext{
I4. "The initial planning for refashioning the Museum Island into a "temple city of art and culture from 6,00o years of human history" commenced shortly after reunification. » (Junod et al., 20I2, p. 294).
} 
des arts de l'Islam ${ }^{15}$. Ensuite, ces artefacts ont fait partie du département des Antiquités créé en 1807. Après l'arrivée de Sir Augustus Wollaston Franks (18261897) au musée en 1851, le département des Antiquités est divisé, en 1860, en trois départements en fonction de l'emplacement géographique et la matière de l'objet (Junod et al., 2012). Ainsi sont créés les départements d'Antiquités gréco-romaines, Monnaies et médailles et Antiquités orientales. Dès lors, il est intéressant de souligner l'intérêt de Wollaston Franks de collectionner les objets des arts de l'Islam afin de retracer le lien entre ces objets et les arts décoratifs en Europe. Il semble nécessaire de noter que le South Kensington Museum créé en 1852, est contemporain de l'époque de Wollaston Franks. En effet, la création du South Kensington Museum a mis en évidence la valeur esthétique d'ornementation dans les arts de l'Islam afin d'améliorer les reproductions et le design chez les artisans anglais et européens. Il semblerait que cette création muséale ait permis une recherche sur la connexion entre les arts de l'Islam et les arts décoratifs en Europe. Cependant, la collection des arts de l'Islam au British Museum n'était pas envisagée comme une collection à part entière mais bien pour ce qu'elle permettait de comprendre de l'évolution historique des formes artistiques européennes (Abdallah, 2010, p. 97). Wollaston Franks a attribué les arts de l'Islam à une zone géographique reliant l'Europe à l'Asie et à l'Afrique à la période entre l'Antiquité et la Renaissance européenne, le Moyen-Âge. La décision est prise en 1984 d'établir la galerie John Addis of the Islamic world, ouverte au public en 1989. Depuis 2018, l'Albukhary Foundation Gallery of the Islamic world a succédé à la galerie John Addis pour l'exposition des arts de l'Islam. Ce changement a effectivement renforcé l'intégration des arts de l'Islam dans le parcours du musée et leur présentation dans les salles. Il situe les arts de l'Islam dans le parcours de l'Europe de l'an 300. De ce fait, la mise en évidence s'établit sur la relation du monde islamique avec l'Europe, de l'Antiquité à nos jours.

L'objectif de la nouvelle exposition permanente au British Museum est de refléter essentiellement la culture matérielle des sociétés originaires des régions où l'islam joue un rôle majeur, voire un rôle unique, dans la culture. La galerie concentre ainsi son approche sur l'exposition des objets qui reflètent l'activité humaine, c'est-à-dire, des objets de la vie quotidienne plutôt que des œuvres d'art. Contrairement au Louvre, où la majorité des objets présentés ont été fabriqués pour les élites dirigeantes, l'objectif du British Museum est d'éviter une telle approche qui offre une image déformée des arts de l'Islam (Akhbarnia et al., 2018). Il s'agit de mettre en évidence tout le spectre de la production allant des ateliers impériaux aux industries artisanales populaires. De plus, en intégrant l'art contemporain dans son discours à travers des œuvres mettant en valeur la calligraphie arabe, le British Museum offre un regard sur les enjeux de

I5. La collection de Sir Hans Sloane est constituée de 79575 objets composés des plantes, fossiles, minéraux, spécimens zoologiques, anatomiques et pathologiques, antiquités et curiosités artificielles, estampes, dessins et monnaies, livres et manuscrits. Parmi ces objets, il se trouve une amulette de quartz gravée de versets du Coran (Abdallah, 20Io). 
la production culturelle contemporaine permettant ainsi d'ajouter une nouvelle dimension de réflexion sur les représentations contemporaines des cultures musulmanes. Dès lors, l'approche de la galerie, en mettant l'accent notamment sur l'anthropologie des sociétés musulmanes, diffère des autres musées. Cette approche aborde ensuite l'art et l'archéologie comme partie intégrante de la culture matérielle islamique.

\section{L'interculturalité selon les arts de l'Islam}

Alors que l'exposition des arts de l'Islam au British Museum n'occupe que deux galeries dans un parcours plus large sur l'évolution de l'art européen, nous remarquons que l'attention du musée diffère de celle du musée du Louvre ou $\mathrm{du}$ Pergamonmuseum. Tout d'abord, tous les trois cherchent à évoquer la relation entre les arts de l'Islam et l'art en Europe, ainsi que le développement des arts de l'Islam. En effet, les trois musées situent les arts de l'Islam dans un contexte chronologique régional, continental et mondial plus vaste, en contraste avec les autres arts dans le monde, notamment l'art occidental. Il est nécessaire de noter que beaucoup d'objets des arts de l'Islam partagent des histoires qui peuvent les situer dans un discours occidental ou islamique, comme par exemple le baptistère de Saint-Louis (Syrie, 1320-1340) au musée du Louvre, ou la corne en ivoire fatimide (Sicile, XIIe-XIIIe siècle) au Pergamonmuseum. Ainsi, la mise en contexte des objets joue un rôle primordial dans la transmission du message. En effet, les trois musées évitent d'isoler leur présentation des arts de l'Islam de l'ensemble des collections au musée, et tous les trois cherchent à montrer la complexité et la relation interculturelle des civilisations de l'Orient et de l'Occident. Dès lors, ils proposent dans leurs approches un traitement interculturel des arts de l'Islam afin de mettre en évidence les points de rencontre entre le monde islamique et l'Europe. Cela correspond effectivement au message porté par le musée. La mise en contexte peut également ajouter une autre dimension à l'exposition. Par exemple, les objets sont souvent exposés dans une ambiance séculaire qui n'empêche cependant pas l'exposition de chercher à évoquer un environnement religieux, comme au Pergamonmuseum où l'une des salles comprend un mihrab (une niche de prière) mis à côté d'un kursi (un lutrin pour le Coran) en bois. Ils sont ainsi exposés à proximité de la vitrine qui parle de madrasa en tant que lieu d'enseignement de science religieuse sous les Seldjoukides (1071-1307). La mise en contexte dans cette partie de l'exposition évoque une aire religieuse et spirituelle et lui donne une autre dimension.

\section{Scénographie décorative ou orientaliste ?}

Dans le cas du Pergamonmuseum, des éléments architecturaux sont mis en place pour représenter une salle soutenue par deux colonnes sur lesquelles se trouvent trois voutes en berceau. La salle est équipée d'une fenêtre munie d'une colonne qui soutient deux voutes décorées de motifs végétaux et géométriques entrelacés et abstraits, l'arabesque. Il convient de noter que ces éléments architecturaux sont fabriqués par le musée afin de créer une scénographie d'ambiance qui sert à la 
mise en contexte du contenu de la salle. En effet, la salle comprend des objets architecturaux authentiques comme une niche en bois et un mihrab ainsi que des objets sensibles à la lumière du jour comme un tapis et une marionnette de théâtre d'ombres. Les interventions scénographiques peuvent servir la fonction de conservation puisquelles protègent les ouvres de la lumière du jour. Un autre exemple se trouve dans une salle où une reconstitution en plâtre monochrome de l'iwan (une salle ouverte à la voûte en berceau) de Taq-e Kisra sert à exposer des éléments architecturaux authentiques et offre aux visiteurs une meilleure compréhension des composants d'un iwan. Dès lors, la scénographie au Pergamonmuseum n'est pas seulement une scénographie d'ambiance mais aide également à illustrer le message porté par le musée sur le développement de l'architecture dans les arts de l'Islam. Il est cependant intéressant de se rappeler que de telles constructions ont fait partie du projet impérialiste du musée d'art décoratif, le South Kensington Museum à Londres. Contrairement à ce musée, le Pergamonmuseum se focalise principalement dans son approche sur l'archéologie et ses constructions scénographiques reflètent ses propos.

Le département des arts de l'Islam au musée du Louvre se caractérise quant à lui par une intervention architecturale contemporaine qui désigne un tapis volant ou dans les mots des architectes et des muséographes Bellini, Ricciotti et Pierard, un voile lumineux (Makariou, 2012, pp. 21-22). Sur le niveau architectural, l'idée perçue ici est de concevoir un échange de regards entre les arts de l'Islam et l'architecture environnante mais cela semble aussi évoquer une rupture avec la représentation des arts de l'Islam et le reste du musée. De plus, la description du toit de la cour en tant que voile peut faire référence aux coutumes des femmes musulmanes et faire appel à une identité ethnique et religieuse qui n'est pas traitée au Louvre. Si cette intervention contemporaine attire beaucoup de visiteurs et les invite à se rendre au musée, elle ne sert pas à refléter le contenu de l'exposition. Cependant, elle remplit une fonction de conservation puisqu'elle filtre la lumière du jour et protège les objets exposés. Le contraste est aussi marqué en passant au niveau du sous-sol qui désigne un espace très sombre ou un océan noir, contrairement à l'espace lumineux au rez-de-chaussée. Jean-Gabriel Leturcq (2012) voit dans la présentation des arts de l'Islam au musée du Louvre une conception néo-orientaliste. En effet, les muséographes ont choisi de faire une représentation de type forêt des vitrines transparentes dans la salle à espace ouvert au sous-sol, ce qui la différencie du reste du musée où les salles sont en majorité de petites salles fusionnées. Dès lors, la scénographie au département des arts de l'Islam contribue à la création d'un espace d'attraction des visiteurs bien que l'architecture contemporaine et les vitrines de haute technologie servent à remplir certaines fonctions de conservation donnant une identité propre au musée.

Enfin, le British Museum a choisi une approche différente de celle du musée du Louvre et du Pergamonmuseum. Alors que son exposition des arts de l'Islam est considérée comme la plus récente, elle ne se caractérise ni par une intervention contemporaine, ni par des reconstitutions d'éléments architecturaux créant une 
scénographie d'ambiance. En effet, son exposition occupe deux galeries du XIXe siècle identiques aux galeries adjacentes mettant en évidence la continuité de son parcours sur le développement de l'art européen. Cependant, il s'y trouve deux fenêtres couvertes par un élément décoratif qui pourrait faire référence à l'architecture islamique connue en tant que moucharabieh. Cet élément participe essentiellement à filtrer la lumière traversant la fenêtre et protège les œuvres de la lumière du jour. Alors que le message de l'exposition est illustré à travers l'exposition des artefacts réels, il ne nécessite aucune pratique de reconstitution des éléments architecturaux complémentaires. Nous pouvons remarquer que la scénographie employée dans les galeries au British Museum est conçue afin de relier l'ensemble des galeries du parcours sur l'art européen.

\section{Conclusion}

Dans cette recherche, nous avons proposé une définition des arts de l'Islam qui pourrait refléter ainsi certains aspects des problématiques de la discipline. Les arts de l'Islam ont été influencés par le contexte politique, sociétal et économique tout au long des XIX ${ }^{e}$ et $\mathrm{XX}^{\mathrm{e}}$ siècles. Au travers des représentations auxquelles ils ont été soumis, ils ont constitué des moyens d'inscrire et de diffuser les messages du pouvoir, tout en étant conditionnés par les représentations authentiques influencées par l'imaginaire collectif occidental. Aujourd'hui, ces représentations marquent une continuité du regard occidental sur la culture de l'Autre, et sont conçues pour la grande majorité par et pour les occidentaux. Par exemple, le Musée royal de l'Afrique centrale à Tervuren représente cette zone géographique en tant que sujet d'étude où la diversité culturelle et ethnique est mise en valeur tout comme l'évolution urbaine et la biodiversité contextualisées par une mise en scène décorative pour constituer un lieu d'attraction. Dans cette perspective, le choix et la manière de représenter l'Autre et de se représenter soi-même entrent dans une dynamique de pouvoir déséquilibrée qui joue en faveur de l'Occident, notamment en ce qui concerne le choix et la manière de se représenter dans les pays des autres comme le souligne l'exemple du musée du Louvre à Abu Dhabi.

De plus, la création de la notion d'Orient selon Edward Said (2005) sert à marquer la différence entre les civilisations islamiques et occidentales. Pourrions-nous cependant affirmer que l'ouverture culturelle sur le monde islamique à travers les nouvelles expositions des arts de l'Islam en Europe est censée construire un pont entre les civilisations contemporaines? La réponse à cette question est effectivement illustrée dans le discours historique et muséographique des musées. L'examen de la médiation entre collections, institutions et espace public permet d'analyser les enjeux politiques de l'exposition des arts de l'Islam. Nous avons remarqué que les trois musées intègrent les arts de l'Islam dans un discours plus large sur les relations interculturelles en appuyant sur les points de rencontre entre l'Occident et l'Orient. La création de ce rapport inter-civilisationnel est nécessaire lorsque l'on expose les cultures de l'Autre en prenant en compte une scénographie adéquate qui servira à l'orientation 
des visiteurs et à la médiation du contenu de l'exposition. C'est le cas de l'exposition des arts de l'Islam au British Museum où l'histoire des communautés musulmanes est racontée à travers les récits d'artefacts. En effet, le British Museum a modifié son champ d'action afin de présenter les cultures matérielles des civilisations : cette approche peut certainement constituer une réflexion nécessaire ainsi qu'une solution aux problématiques du terme et de la discipline des arts de l'Islam. The Albukhary Foundation Gallery of the Islamic world au British Museum emploie également une scénographie correspondant à la conception des galeries avoisinantes renforçant ainsi la cohésion entre les collections du musée et l'ensemble de son parcours sur le développement des arts en Europe. Elle constitue donc le modèle de référence pour les expositions des arts de l'Islam en Occident.

\section{Références}

Abdallah, M. (2010). Exposer l' «art contemporain du Moyen-Orient»: le British Museum face à ses collections. Intermédialités: Histoire et théorie des arts, des lettres et des techniques, 15, 91-104.

Akhbarnia, L., Porter, V., Suleman, F., Greenwood, W., Klink-hoppe, Z., \& Mérat, A. (2018). The Islamic world: A history in objects [Exhibition catalogue]. Thames \& Hudson.

Allen, T. (1988). Five essays on Islamic art. Solipsist Press.

Ali, W. (1997). Modern Islamic art: Development and continuity. University Press of Florida.

Amselle, J. (2016). Le musée exposé. Lignes.

Barringer, T. (1998). The South Kensington Museum and the colonial project. In T. Barringer \& T. Flynn, Colonialism and the object: Empire, material culture and the museum, (pp. 11-27). Routledge.

Blair, S. S., \& Bloom, J. M. (2003). The mirage of Islamic art: Reflections on the study of an unwieldy field. The Art Bulletin, 85(1), 152-184.

Çelik, Z. (1992). Displaying the Orient. Architecture of Islam at nineteenth-century world's fairs. University of California Press.

Conway, M. D. (1882). Travels in South Kensington with notes on decorative art and architecture in England. Harper \& Brothers.

Crill, R., \& Stanley, T. (2006). The making of the Jameel Gallery of Islamic Art at the Victoria and Albert Museum [Exhibition catalogue]. Victoria \& Albert Museum.

Duthie, E. (2011). The British Museum: An imperial museum in a post-imperial world. Public History Review, 18,12-24. 
Fulco, D. (2017). Display of Islamic art in Vienna and Paris: Imperial politics and exoticism at the Weltausstellung and Exposition Universelle. MDCCC 1800, 6(1), 51-66.

Grabar, O. (1973). The formation of Islamic art. Yale University Press.

Grabar, O. (1983). Reflections on the study of Islamic art. Muqarnas, 1, 1-14.

Greenwood, W. (2019, February 26). Albukhary Foundation Curator of the Islamic World. The British Museum. https://blog.britishmuseum.org/theislamic-world-the-big-themes/

Junod, B., Khalil, G., Weber, S., \& Wolf, G. (2012). Islamic art and the museum: Approaches to art and archeology in the Muslim world in the twenty-first century. Saqi Books.

Labrusse, R. (Ed.). (2007). Pure décor? Arts de l'Islam, regards du XIXe siècle [Exhibition catalogue]. Musée du Louvre.

Leprun, S. (1986). Le théâtre des colonies: Scénographie, acteurs et discours de limaginaire dans les expositions, 1855-1937. L'Harmattan.

Leturcq, J.-G. (2012, September 2). Inventing Islamic Art (2): The French connection. Jean-Gabriel Leturcq. https://leturcq.wordpress.com/2012/09/21/ inventing-islamic-2/

Makariou, S. (Ed.). (2012). Les arts de l'Islam au Musée du Louvre [Exhibition catalogue]. Musée du Louvre.

Maussen, M. (2007). Islamic presence and mosque establishment in France: Colonialism, arrangements for guestworkers and citizenship. Journal of Ethnic and Migration Studies, 33(6), 981-1002.

Pinna, G. (2011). Islam in the arts: New museography of Islamic arts. Museum International, 63(3-4), 104-114.

Rabbat, N. (2004). Islamic architecture as a field of historical enquiry. Architectural Design 74(6), 18-23.

Robertson, B. (2004). The South Kensington Museum in context: An alternative history. Museum and Society, 2(1), 1-14.

Roxburgh, D. J. (2000). Au bonheur des amateurs: Collecting and exhibiting Islamic art, ca. 1880-1910. In L. Komaroff(Ed.), Ars Orientalis: Exhibiting the Middle East. Collections and perceptions of Islamic Art, 30, (pp. 9-38), University of Michigan.

Said, E. W. (2005). L'orientalisme. L'Orient créé par l'Occident. (C. Malamoud, Trans.). Édition du Seuil. (Original work published 1978)

Shalem, A. (2012). What do we mean when we say 'Islamic art'? A plea for a critical rewriting of the history of the arts of Islam. Journal of Art Historiography, 6, 1-18. 